\title{
An improved CMOS-based inductor simulator with simplified structure for low-frequency applications
}

\author{
Longjie Zhong ${ }^{1} \cdot$ Xinquan Lai $^{1} \cdot$ Donglai $\mathrm{Xu}^{2,3} \cdot$ Michael Short $^{2} \cdot$ Bing Yuan $^{1} \cdot$ \\ Zeyu Wang ${ }^{1}$
}

Published online: 30 May 2016

(C) The Author(s) 2016. This article is published with open access at Springerlink.com

\begin{abstract}
In this paper, an improved inductor simulator structure is presented, which can be configured as either grounded or floating inductor simulator with low component count. To achieve simplified structure, inductor simulator circuits are designed using a minimal number of transistors and small capacitance, rather than the complex components/modules such as current convey and operational trans-conductance amplifier which are traditionally used. The simulation results based on $0.5 \mu \mathrm{m}$ CMOS process parameters show that the proposed structure is able to produce a broad range of inductance values and compared to other similar structures, it provides wider operational frequency bandwidth for the same or comparable inductance value. Furthermore, the structure can be implemented with much smaller chip area using a small capacitance in the circuit, but at the price that it has a higher minimum operational frequency compared to other structures.
\end{abstract}

Keywords Grounded inductor simulator · Floating inductor simulator - Active inductor . CMOS integrated circuits . MOSFET $\cdot$ Operational frequency

\section{Introduction}

Inductance is a vital component for many analog and mixedsignal circuits and systems. Large inductances are often

Longjie Zhong

zhong.longjie@163.com

1 Institute of Electronic CAD, Xidian University, Xi' an, China

2 School of Science and Engineering, Teesside University, Middlesbrough TS1 3BA, UK

3 School of Electrical and Electronic Engineering, Wuhan Polytechnic University, Wuhan, China needed if operational frequency of a circuit is not very high, but they are difficult to be integrated into an integrated circuit (IC) due to the large chip area required. There have been a number of attempts to develop inductor simulators, which can perform the analog function of inductance [1-12]. The most commonly used structures of these inductor simulators are composed of multiple passive components and complicated operational modules such as current conveyor (CC) [2-5], current feedback operational amplifier (CFOA) [6], operational trans-conductance amplifier (OTA) [7], current backward trans-conductance amplifier (CBTA) [8] and current differencing buffered amplifier (CDBA) [9]. This is because that they attempt to achieve functional flexibility, i.e., to be reconfigured to form other circuit functions such as frequency dependent negative resistor (FDNR), while aiming at low-frequency applications. Other structures [10-12] use fewer and less complicated components to be structurally simple and to minimize the effect of parasitic parameters. However, these are primarily used for high-frequency or $\mathrm{RF}$ applications. In this paper, an improved structure for grounded inductor composed of only five active components and one capacitor for low-frequency applications is proposed. Compared to the structure in [1] which was proposed for the same purpose, this structure is further simplified and is able to simulate the same or comparable inductance value with smaller capacitor and wider operational frequency bandwidth. In addition, by adding only three more transistors to the structure, the grounded inductor simulator can be easily upgraded to floating inductor simulator.

The rest of this paper is organized as follows: Section 2 presents the process of designing inductor simulator. Section 3 shows simulation results and analysis of the proposed inductor simulators. Finally, conclusions are drawn in Sect. 4. 


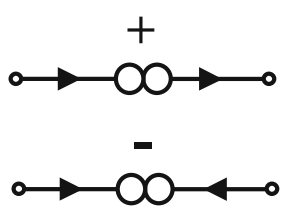

(a)

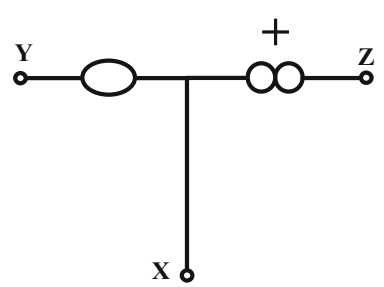

(c) (b)

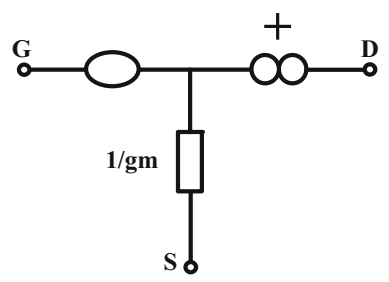

(d)

Fig. 1 a Nullator, b norator, c second generation current conveyor (CCII), $\mathbf{d}$ nullator-norator model of MOSFET

\section{Proposed inductor simulator}

To achieve simplified structure and simulate a range of inductance values at low operational frequency, MOSFET is to be used as prime component rather than CC or OTA. The design method is based on the nullator-norator technique. A nullator represents a port that has no potential difference across its two terminals and has no current flowing into or out of it, as shown in Fig. 1a. A norator represents a port that has arbitrary current flowing through and has arbitrary potential difference across its two terminals, as shown in Fig. 1b. The nullatornorator models of second generation current conveyor (CCII) and MOSFET are shown in Fig. 1c, d [5]. Figure 1 illustrates clearly the structural similarity between CCII and MOSFET, which suggests that instead of CCII, MOSFET may be used to construct an inductor simulator.

There are two key components that are used to build the proposed inductor simulator, MOSFET and capacitor. MOSFET is used to convert voltage signal into current signal. The capacitor is used to emulate voltage-current characteristic of inductor, i.e., [13]

$$
\begin{aligned}
& Z_{\mathrm{C}}=\frac{V_{\mathrm{C}}}{I_{\mathrm{C}}}=\frac{1}{j \omega X_{\mathrm{C}}} \\
& Z_{\mathrm{L}}=\frac{V_{\mathrm{sim}}}{I_{\mathrm{sim}}}=j \omega X_{\mathrm{L}}
\end{aligned}
$$

where $V_{\mathrm{C}}$ and $I_{\mathrm{C}}$ are the voltage and the current applied to capacitor, $V_{\text {sim }}$ and $I_{\text {sim }}$ are the voltage and the current applied to inductor, $X_{\mathrm{C}}$ is the value of the capacitor, $X_{\mathrm{L}}$ is the value of the inductor, $\omega$ is operational frequency, and $j$ is the imaginary unit representing 90 -degree phase shift. It is

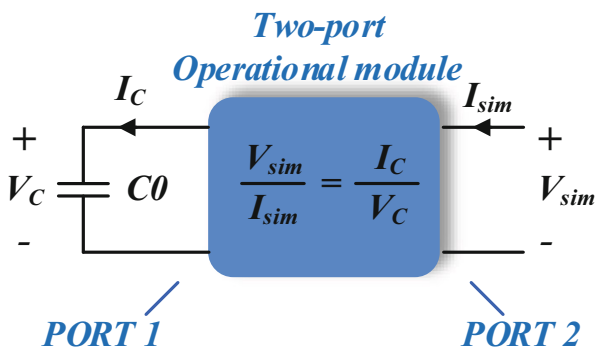

Fig. 2 Block diagram of inductor simulator

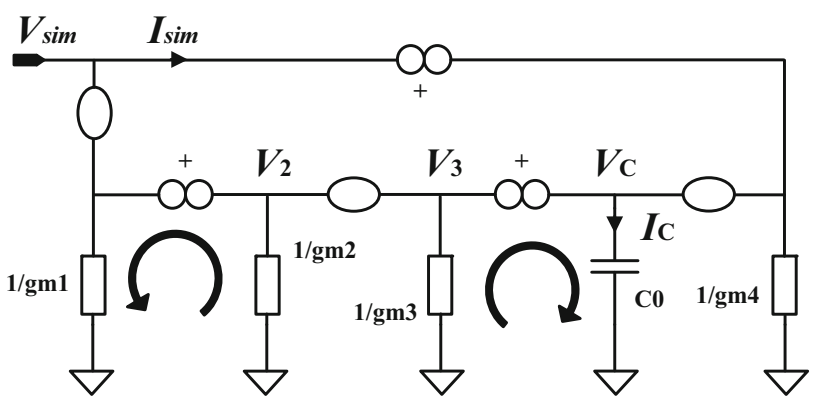

Fig. 3 Nullator-norator structure of inductor simulator

obvious from Eq. (1) that if $X_{\mathrm{C}}=X_{\mathrm{L}}$, the impedance of the capacitor is reciprocal of the inductor's impedance.

To realize simulation of inductor, an inductor simulator is constructed here using a two-port operational module, as shown in Fig. 2. One port (PORT 1) of the module connects to a capacitor $C 0$. The other port (PORT 2) connects to an arbitrary external circuit. The function of this operational module is to make $V_{\text {sim }} / I_{\text {sim }}$ equal to $I_{C} / V_{C}$, so that the impedance of the simulated inductor $\left(V_{\mathrm{sim}} / I_{\mathrm{sim}}\right)$ is the same as the reciprocal of the impedance of the capacitor $\mathrm{C} 0$. It operates as follows: Once the voltage $V_{\text {sim }}$ is applied onto PORT 2, the current $I_{\mathrm{C}}$ that is proportional to $V_{\text {sim }}$ is generated and fed into the capacitor $\mathrm{C} 0$ in PORT 1 , therefore producing the voltage $V_{\mathrm{C}}$ across the capacitor. Then from the $V_{\mathrm{C}}$, the current $I_{\text {sim }}$ that is proportional to $V_{\mathrm{C}}$ is generated and fed back into PORT 2.

Following the working explained above, the nullatornorator structure of the inductor simulator is acquired, as shown in Fig. 3.

The relationship between the port voltage $V_{\text {sim }}$ and the port current $I_{\text {sim }}$ can be deduced as [14]

$\frac{I_{\text {sim }}}{V_{\text {sim }}}=\frac{g_{m 1} g_{m 3} g_{m 4}}{g_{m 2}} \frac{V_{\mathrm{C}}}{I_{\mathrm{C}}}=\frac{1}{j \omega L_{\text {sim }}}$,

where $L_{\text {sim }}=\frac{g_{m 2}}{g_{m 1} g_{m 3} g_{m 4}} C 0$ is the inductor to be simulated through the capacitor $\mathrm{C} 0$. By replacing the nullatornorator pairs in Fig. 3 with MOSFETs, we can obtain the grounded inductor simulator circuit, as shown in Fig. 4. The $g m 1, g m 2, g m 3$, and $g m 4$ in Fig. 3 are trans-conductances 


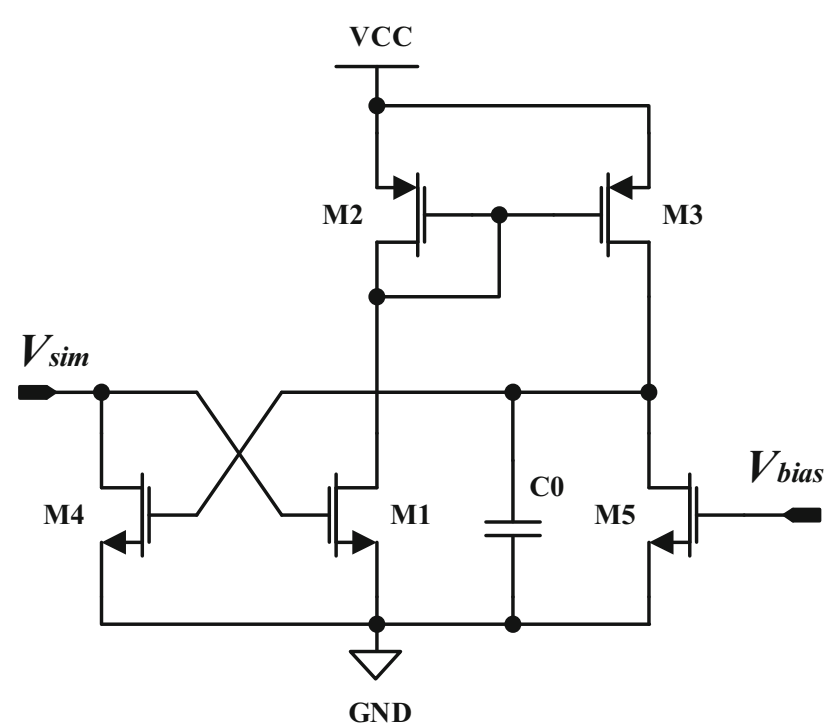

Fig. 4 Grounded inductor simulator circuit

of the MOSFETs M1, M2, M3, and M4 in Fig. 4, respectively. The MOSFET M5 is to provide a current bias for the circuit.

In order to consider the main parasitic parameters that will affect the frequency response of the circuit, Eq. (2) needs to be modified by taking parasitic capacitance and output resistance of MOSFETs into account [14], and then it becomes

$$
\begin{aligned}
\frac{I_{\mathrm{sim}}}{V_{\mathrm{sim}}}= & G_{m} R_{\mathrm{O} 35} \frac{1}{1+j \frac{\omega}{\omega_{0}}} \frac{1}{1+j \frac{\omega}{\omega_{1}}}+\frac{1}{R_{\mathrm{O} 4}} \\
& +j \omega\left(C_{\mathrm{gs} 1}+C_{\mathrm{ds} 4}\right)
\end{aligned}
$$

where $G_{m}=\frac{g_{m 1} g_{m 3} g_{m 4}}{g_{m 2}}$ is the open loop trans-conductance, $R_{\mathrm{O} 35}=R_{\mathrm{O} 3} \mid R_{\mathrm{O} 5}{ }_{m}$ is the resultant resistance of the output resistance of $\mathrm{M} 3\left(R_{\mathrm{O} 3}\right)$ and the output resistance of M5 $\left(R_{\mathrm{O} 5}\right)$ in parallel connection, $R_{\mathrm{O} 4}$ is the output resistance of M4, $C_{\mathrm{gs}}$ and $C_{\mathrm{ds}}$ are the gate-source parasitic capacitance and the drain-source parasitic capacitance of MOSFET, respectively, and $\omega_{0}=g_{m 2} /\left(C_{\mathrm{gs} 2}+C_{\mathrm{gs} 3}+C_{\mathrm{ds} 2}+C_{\mathrm{ds} 1}\right)$ and $\omega_{1}=1 / R_{035} C 0$ are the two poles of the open loop transfer function. The $\omega_{0}$ is always a very high-frequency pole, which is normally negligible.

According to the conventional calculations [14], if the frequency $\omega>10 \omega_{1}$, Eq. (3) can be simplified as

$\frac{I_{\text {sim }}}{V_{\text {sim }}}=\frac{1}{j \omega L_{\text {sim }}}+\frac{1}{R_{\text {sim }}}+j \omega C_{\text {sim }}$,

where

$$
\begin{aligned}
L_{\text {sim }} & =\frac{g_{m 2}}{g_{m 1} g_{m 3} g_{m 4}} C 0, \\
R_{\text {sim }} & =R_{O 4}, \\
C_{\text {sim }} & =C_{\mathrm{gs} 1}+C_{\mathrm{ds} 4} .
\end{aligned}
$$

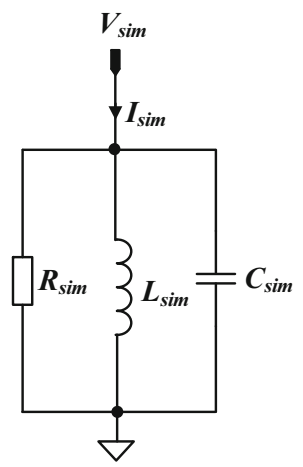

(a) Inductor equivalent circuit with high frequency (b) Inductor equivalent circuit with low frequency

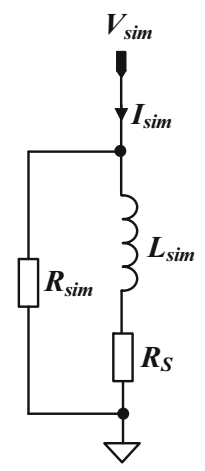

Fig. 5 Equivalent circuits of grounded inductor simulator

If the frequency $\omega<10 \omega_{1}$, Eq. (3) can be simplified as

$$
\frac{I_{\text {sim }}}{V_{\text {sim }}}=\frac{1}{R_{\mathrm{s}}+j \omega L_{\text {sim }}}+\frac{1}{R_{\text {sim }}},
$$

where

$$
\begin{aligned}
& L_{\text {sim }}=\frac{g_{m 2}}{g_{m 1} g_{m 3} g_{m 4}} C 0, \\
& R_{\mathrm{s}}=\frac{g_{m 1} g_{m 3} g_{m 4}}{g_{m 2}} R_{\mathrm{O} 35}, \\
& R_{\mathrm{sim}}=R_{\mathrm{O} 4} .
\end{aligned}
$$

Equations (4) and (5) mean that the circuit in Fig. 4 can be simplified to one of the two equivalent circuits shown in Fig. 5. Figure 5a shows the equivalent inductor simulator circuit operating at high frequency, which is derived through Eq. (4). Figure $5 \mathrm{~b}$ shows the equivalent inductor simulator circuit operating at low frequency, which is derived through Eq. (5).

From Eqs. (4) and (5) as well as Fig. 5, it is easy to understand that the frequency range of equivalent circuit is determined by $C_{\mathrm{sim}}$ at high frequency and by $R_{\mathrm{S}}$ at low frequency. This is because that the $C_{\text {sim }}$ together with $L_{\text {sim }}$ will form a double-pole point (or resonation point), which prevents magnitude response from keeping rising up, and that the $R_{\mathrm{S}}$ together with $L_{\text {sim }}$ will form a zero point, which prevents magnitude response from keeping going down. Hence, the upper and lower limits of the operational frequency are decided by the following double-pole point $\omega_{\mathrm{D}-\text {-pole }}$ and zero point $\omega$ Zero, respectively:

$$
\begin{aligned}
\omega_{\mathrm{D}-\text { pole }} & =\frac{1}{\sqrt{L_{\text {sim }} C_{\text {sim }}}}, \\
\omega_{\text {Zero }} & =\frac{R_{\mathrm{s}}}{L_{\text {sim }}} .
\end{aligned}
$$

Furthermore, by replacing the $V_{\text {bias }}$ terminal in Fig. 4 with another grounded inductor simulator, a floating inductor sim- 


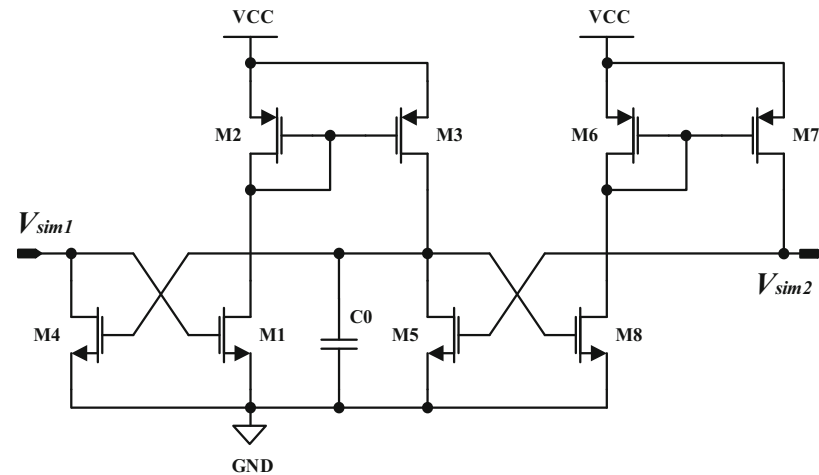

Fig. 6 Floating inductor simulator derived from grounded inductor simulator

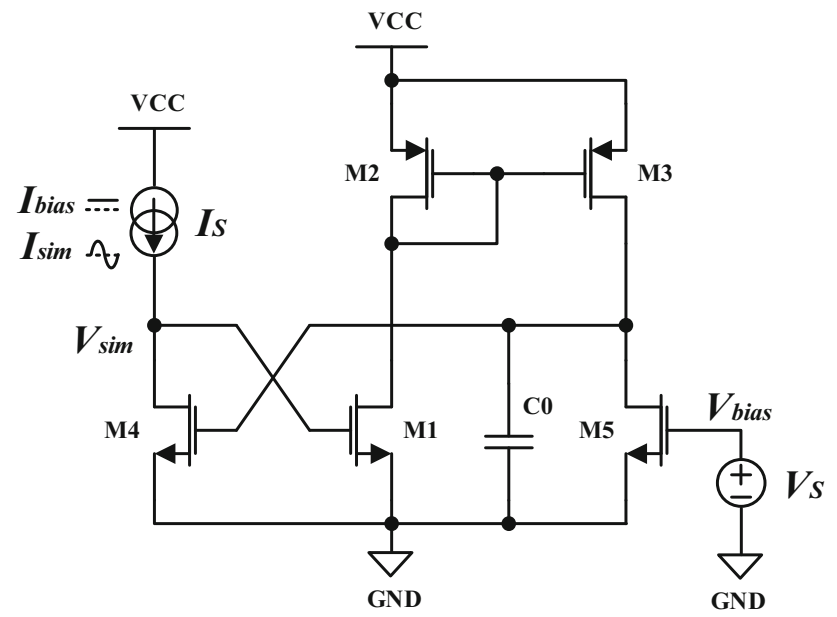

Fig. 7 Configuration for simulations of grounded inductor simulator

ulator can be acquired, as shown in Fig. 6. This floating inductor simulator has the same function and electrical characteristics as the grounded inductor simulator in Fig. 4, but it is more flexible in terms of its applications since both of its terminals can be connected to other circuits.

\section{Simulation results and analysis}

The circuit of Fig. 4 is simulated using the configuration shown in Fig. 7. The terminal $V_{\text {bias }}$ connects to a voltage source $V_{\mathrm{S}}$ to provide DC voltage bias for the MOSFET M5. The terminal $V_{\text {sim }}$ connects to a current source $I_{\mathrm{S}}$ to provide the DC current bias $I_{\text {bias }}$ for the MOSFET M4 and to provide the AC signal excitation $I_{\text {sim }}$ as well. The bulks of NMOS and PMOS transistors are connected to the ground GND and the power supply VCC, respectively. The simulations are performed using SPICE based on $0.5 \mu \mathrm{m}$ CMOS process BSIM3v3 model (the threshold voltages of NMOS and PMOS are $V_{\mathrm{TN} 0}=0.7619 \mathrm{~V}$ and $V_{\mathrm{TP} 0}=-0.9570$ $\mathrm{V}$, respectively; the electron mobility and hole mobility are $u_{0 N}=861.083 \mathrm{~cm}^{2} / \mathrm{Vs}$ and $u_{0 P}=568.314 \mathrm{~cm}^{2} / \mathrm{V} \mathrm{s}$,

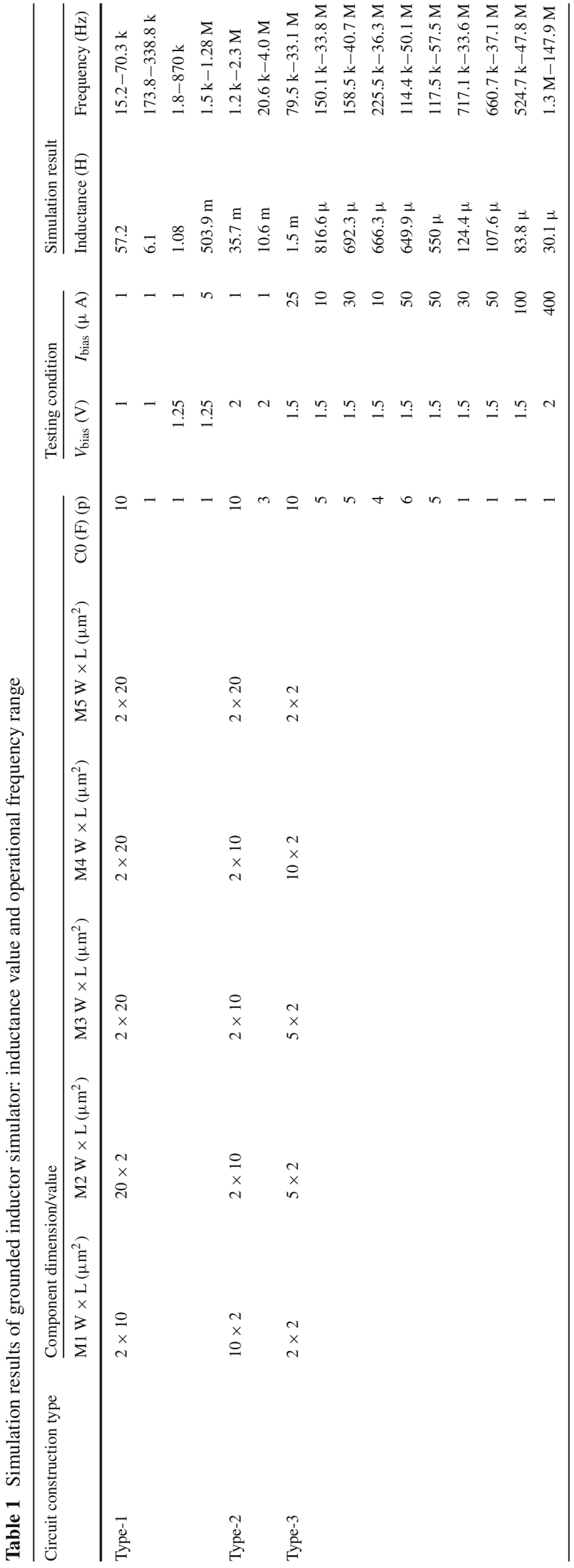




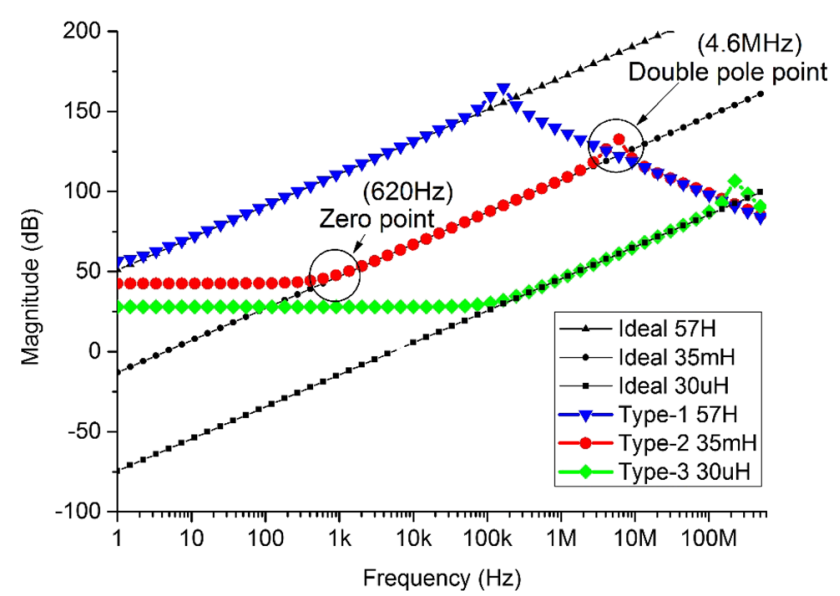

Fig. 8 Magnitude responses of each type compared to ideal inductor

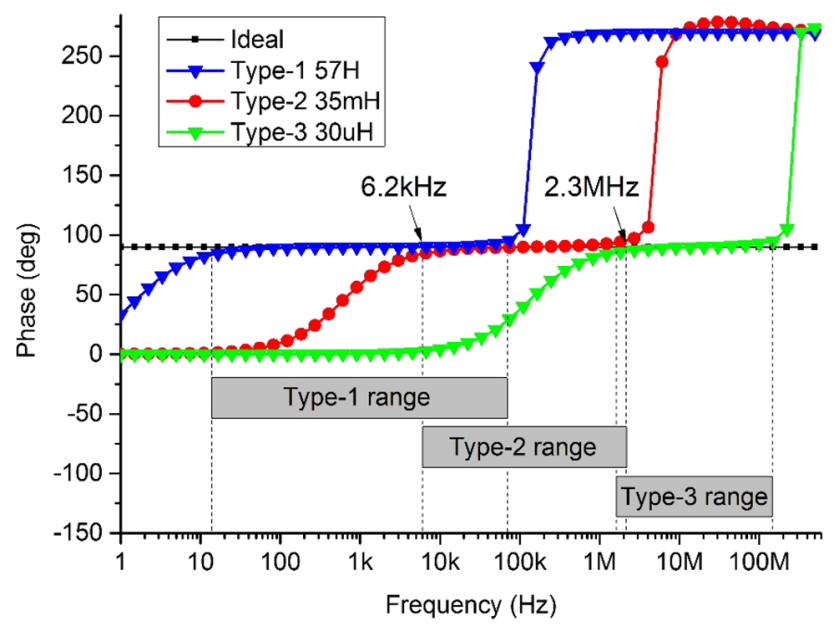

Fig. 9 Phase responses of each type compared to ideal inductor

respectively; the thickness of gate oxide is $T_{\mathrm{OX}}=25 \mathrm{~nm}$ ). The dimensions of the CMOS transistors (M1-M5) used in the implementation are given in Table 1 . To test the functionality of the circuit in Fig. 4, three types of constructions of the circuit with different sizes of components (Type-1, Type-2, and Type-3) are simulated, as shown in the table, so that a variety of inductor values with their corresponding operational frequency ranges can be produced.

The floating inductor simulator in Fig. 6 has the same simulation results as the grounded inductor simulator in Fig. 4, with the transistors M6-M8 having the same dimensions as the transistors M3-M5.

From the simulation results, it is obvious that compared to an ideal inductor which has no frequency restriction, the simulated inductors work within certain limited frequency range. Taken the Type-2 $(35 \mathrm{mH})$ of the circuit as an example, the magnitude responses of the frequency domain simulations in Fig. 8 show that the circuit has a zero point at $620 \mathrm{~Hz}$ and a double-pole point at $4.6 \mathrm{MHz}$, which are determined by $R_{\mathrm{S}}$ and $C_{\text {sim }}$, respectively. This is in accordance with the theo-

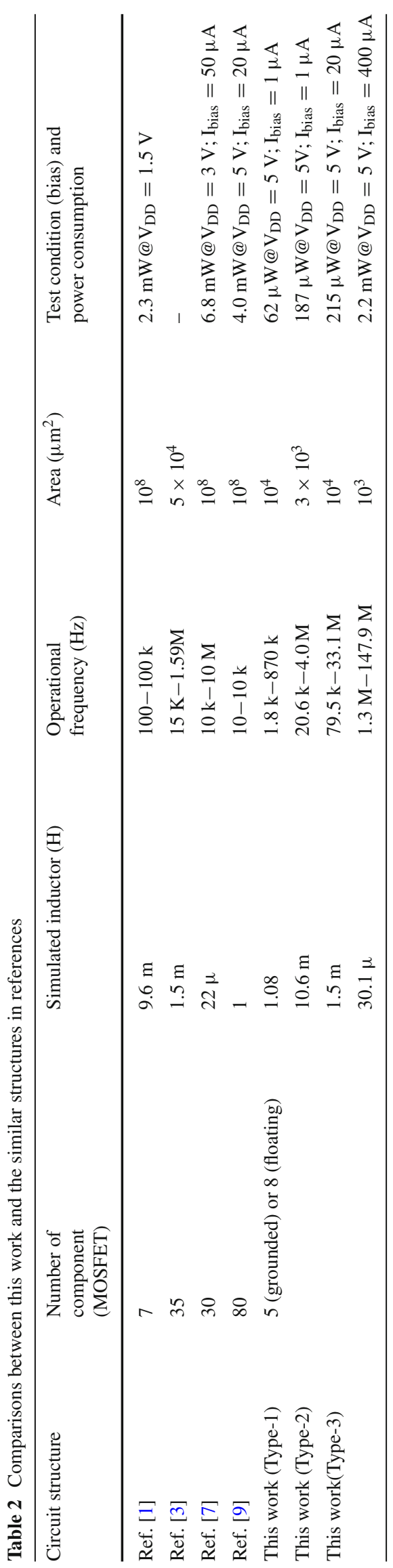


retical analysis of the frequency limits described in Sect. 2 . The phase responses of the frequency domain simulations are given in Fig. 9, which shows that the zero point of $620 \mathrm{~Hz}$ and the double-pole point of $4.6 \mathrm{MHz}$ yield an actual working frequency range from $6.2 \mathrm{kHz}$ ( $10 \omega_{\text {Zero }}$ ) to $2.3 \mathrm{MHz}$ (calculated through $R_{\mathrm{S}}$ and $\left.\omega_{\mathrm{D}-\text { pole }}\right)$.

To compare with the similar structures proposed recently $[1,3,7,9]$, Table 2 is compiled, in which the area is calculated by only taking capacitor into account, as it consumes most of the chip area. It is assumed that $1 \mathrm{fF}$ capacitance takes 1 $\mu \mathrm{m}^{2}$ of chip area. The test conditions (bias) and the power consumptions under these conditions are also given.

Table 2 shows that among the similar structures compared, this structure uses the least number of MOSFETs (only 5 for grounded inductor), leading to the simplest circuit. It is capable of simulating a broad range of inductance values that are covered by all other structures. Meanwhile, for the same or comparable inductance value, its operational frequency bandwidth is much wider (e.g., for $1.0 \mathrm{H}$ inductor, Type 1 of this work and Ref. [9] have a bandwidth of $868.2 \mathrm{~Hz}$ and $10 \mathrm{kHz}$, respectively). However, the proposed structure does not operate at as low frequency as others do (e.g., Type 3 of this work and Ref. [3] with the same inductance value of $1.5 \mathrm{mH}$ have a minimum operational frequency of $79.5 \mathrm{~Hz}$ and $15 \mathrm{KHz}$, respectively), since it uses much smaller capacitors (no more than $10 \mathrm{pF}$ ) in order to significantly reduce chip area as shown in the table.

\section{Conclusions}

This paper describes an improved structure for inductor simulator to be used in CMOS integrated circuits for lowfrequency applications. The structure features low component count and use of small capacitance, thus resulting in simplified circuit structure and much reduced chip area. The simulation results demonstrate that this structure not only can produce a broad range of inductance values but also compared to other similar structures, it provides wider operational frequency bandwidth for the same/comparable inductance value. Moreover, the structure is implemented with significantly reduced chip area using a small capacitor in the circuit, but this is at the cost of having a higher minimum operational frequency compared to other structures.

Acknowledgments This work was supported by the Fundamental Research Funds for the Central Universities of China under the Grant Number JB150222.

Open Access This article is distributed under the terms of the Creative Commons Attribution 4.0 International License (http://creativecomm ons.org/licenses/by/4.0/), which permits unrestricted use, distribution, and reproduction in any medium, provided you give appropriate credit to the original author(s) and the source, provide a link to the Creative Commons license, and indicate if changes were made.

\section{References}

1. Minaei, S., Yuce, E.: A simple CMOS-based inductor simulator and frequency performance improvement techniques. AEU Int. J. Electron. Commun. 66(11), 884-891 (2012)

2. Yuce, E., Cicekoglu, O., Minaei, S.: CCII-based grounded to floating immittance converter and a floating inductance simulator. Analog Integr. Circuits Signal Process. 46(3), 287-291 (2006)

3. Metin, B., Herencsar, N., Koton, J.: DCCII based inductance simulator circuit with minimum number of element. In: 23rd International Conference on Radioelektronika (RADIOELEKTRONIKA). pp. 89-91 (2013)

4. El Feki, N.B., Masmoudi, D.S., Derbel, N.: On the frequency compensation of simulated CCII based tunable floating inductance for LC ladder filters applications. In: 2004 International Conference on Electrical, Electronic and Computer Engineering. ICEEC '04. pp. 449-452 (2004)

5. Higashimura, M., Fukui, Y.: Novel method for realizing lossless floating immittance using current conveyors. Electron. Lett. 23(10), 498-499 (1987)

6. Yuce, E.: Novel lossless and lossy grounded inductor simulators consisting of a canonical number of components. Analog Integr. Circuits Signal Process. 59(1), 77-82 (2008)

7. Kwawsibsam, A., Lahiri, A., Jaikla, W.: Conception of simulating grounded negative inductor and implementation using operational transconductance amplifiers. In: 13th International Symposium on Communications and Information Technologies (ISCIT). pp. 347349 (2013)

8. Ayten, U.E., Sagbas, M., Herencsar, N., Koton, J.: Novel floating FDNR, inductor and capacitor simulator using CBTA. In: 34th International Conference on Telecommunications and Signal Processing (TSP). pp. 312-316 (2011)

9. Keskin, A.U., Erhan, H.: CDBA-based synthetic floating inductance circuits with electronic tuning properties. ETRI J. 27, 239-241 (2005)

10. Li, C., Wang, P., Gong, F.: A low-power active inductor with improved Q-factor and its application to phase shifter. Analog Integr. Circuits Signal Process. 66(2), 303-307 (2011)

11. Rafei, M., Mosavi, M.R.: A new $0.25-12.5 \mathrm{GHz}$ high quality factor low-power active inductor using local RC feedback to cancel seriesloss resistance. Arab. J. Forence Eng. 38(11), 3125-3132 (2012)

12. Zito, D., Pepe, D., Fonte, A.: High-frequency CMOS active inductor: design methodology and noise analysis. IEEE Trans. Very Large Scale Integr. Syst. 23, 1123-1136 (2014)

13. Floyd, T.L.: Electronics Fundamentals: Circuits, Devices \& Applications. Prentice Hall Press, Upper Saddle river (2009)

14. Razavi, B.: Design of Analog CMOS Integrated Circuits. McGrawHill, New Delhi (2003) 